\title{
Fluorescence Anisotropy Reduction of Allosteric Aptamer for Sensitive and Specific Protein Signaling
}

\author{
Dapeng Zhang, ${ }^{\dagger}$ Qiang Zhao, ${ }^{\ddagger}$ Bailin Zhao, ${ }^{\dagger}$ and Hailin Wang* ${ }^{*}{ }^{\dagger}$
}

${ }^{\dagger}$ State Key Laboratory of Environmental Chemistry and Ecotoxicology, Research Center for Eco-Environmental Sciences, Chinese Academy of Sciences, Beijing, 100085, P. R. China

${ }^{\ddagger}$ Research Center for Environmental Science and Engineering, Shanxi University, Taiyuan, Shanxi Province, 030006, P. R. China

Supporting Information

ABSTRACT: Real time protein signaling in a complex medium may provide a promising way for high-throughput protein analysis, but it is largely unmet due to the challenge of signal transduction and the interferences of nonspecific binding and high background. Our recent work indicates that a fluorescent aptamer can display a protein bindinginduced reduction of fluorescence anisotropy (FA) (Zhang, D.; Lu, M.; Wang, H. J. Am. Chem. Soc. 2011, 133, 9188-9191), which is exclusively different from a traditionally simplified concept hinting a molecular size increase-induced FA increase. Inspired by this unexpected observation, we describe a novel FA reduction approach for protein signaling. The feasibility of this approach is demonstrated through the assays of a blood protein human $\alpha$-thrombin and an oncoprotein human platelet-derived growth factor B-chain (PDGF-BB) using two screened fluorescent aptamers,

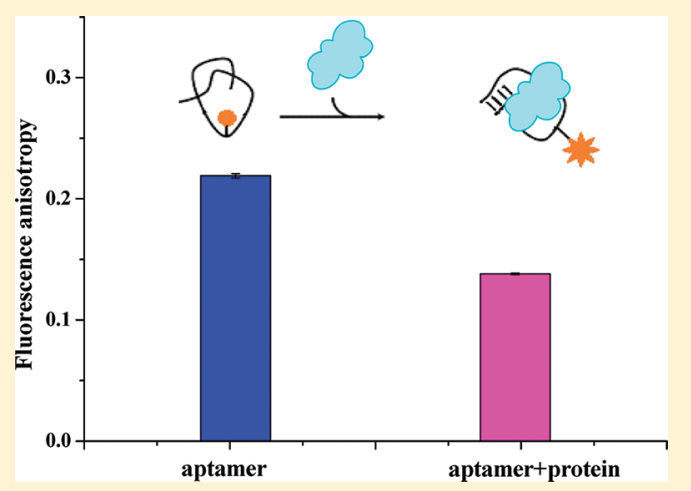
respectively. By the developed FA reduction method, the spiked human $\alpha$-thrombin in diluted serum can be detected at the concentration as low as $250 \mathrm{pM}$. In contrast, in a traditional molecular size-dependent FA assay, the thrombin spiked in diluted serum cannot induce reliable FA change even at a 256 -fold higher concentration (64 nM). The results clearly show that the FA reduction approach has a dramatically enhanced specificity against target protein and high sensitivity in complex medium and is applicable to the no-separation based detection of proteins in biological matrixes.

$\mathrm{V}$ ersatile, high affinity, and high specific nucleic acid aptamers can be selected from an oligonucleotide library, synthesized with normal (and modified) bases, and have tremendous applications in proteomic study, bioanalysis, clinical diagnostics, and therapy. ${ }^{1-10}$ Because of good stability and ease-of-modification, DNA aptamers are favorable affinity ligands for development of real time protein signaling, which may provide a promising way for high-throughput protein analysis. Compared with classic immune-affinity reagents (e.g., IgG $\sim 150 \mathrm{kDa})$, DNA aptamers $(\sim 4.5-30 \mathrm{kDa})$ are relatively small. Thus, the molecular size of an aptamer dramatically increases upon target protein binding, and consequently the tumbling (diffusional rotation) of the aptamer slows down. ${ }^{7,11,12}$ If it is fluorescently labeled with a small dye molecule, the slowly tumbling aptamer displays an increased signal in fluorescence anisotropy (FA). In general, FA is a function of molecular size and increases with increasing molecular size. By taking advantage of the dramatic molecular-size increase in the protein-aptamer binding and single fluorescent labeling of aptamer, the aptamer biosensors can be developed to transduce the binding events into increased FA responses. ${ }^{11-17}$ It is a simple and ideal way for real time protein signaling in homogeneous solution without the need for any separation but probably with a sacrifice of sensitivity, providing the possibility to achieve high-throughput analysis and a large scale of proteomic study. Aptamer-based FA measurements have been exploited for quantitative analysis of macromolecules and cells. ${ }^{11-17}$ In these traditional FA assays, an increase of FA response is produced, depending on an increased molecular size caused by the binding of aptamer to target protein. ${ }^{18}$ Similarly, nonspecific binding raised in complex biological fluids can also display an increased FA response and seriously interfere with the target protein analysis. Therefore, current FA assays meet a great challenge for protein analysis in complex biological fluids. In fact, real time protein signaling, on the basis of other analytical technologies, also remains challenging, ${ }^{19}$ probably due to the interferences resulting from nonspecific binding and high background in a protein-rich matrix, too.

Our recent work indicates that a fluorescent aptamer, labeled by a fluorophore at a selected nucleotide site, can display a reduced FA value in response to the target protein binding, ${ }^{20}$ which is exclusively different from a traditionally simplified concept hinting at molecular size increase-induced FA increase. ${ }^{1-18}$ The observed FA reduction is probably caused by the changes in aptamer conformation and the followed

Received: February 11, 2012

Accepted: March 20, 2012

Published: March 20, 2012 
diminishment of the intramolecular interactions between the labeled fluorophore and the adjacent bases of the aptamer upon the protein binding. Inspired by this unexpected observation, herein we describe an FA reduction approach for protein signaling in complex biological medium achieving an enhanced specificity and lower LOD. The advantage of LOD is primarily a reflection of the fact that nonspecific interactions that interfere with FA have been ameliorated due to FA reduction. Therefore, the approach can overcome the limitation of the traditional molecular size-dependent FA assays and provide the possibilities for practical applications and high-throughput protein analysis.

To demonstrate the principle-of-proof, we first examined the binding of human $\alpha$-thrombin and the aptamer TA29 using homogeneous FA analysis as an example. TA29 has a selected sequence of 5'-AGT CCG TGG TAG GGC AGG TTG GGG TGA CT- 3 ' and may bind human $\alpha$-thrombin in a probable quadruplex/duplex structure with a $K_{\mathrm{d}}$ value of $0.5 \mathrm{nM}^{21}$ We investigated the labeled fluorophores and position on the aptamer, the effects of metal ions, the specific and nonspecific protein binding, and the detection of target protein spiked in biological fluids.

Tetramethylrhodamine (TMR) was chosen for the fluorescent labeling of TA 29 aptamers because of its potential to intramolecularly interact with the $\mathrm{G}$ bases of the aptamers, ${ }^{22-27}$ which is a crucial factor to contribute to the induction of the pursued FA reduction (see later discussion). Two TA29 aptamers (5'-TMR-TA29 and 3'-TMR-TA29, see Table S1 in the Supporting Information) were obtained by the labeling of TA29 at the 5'- and 3'-end, respectively; and one mutant of TA29 (C15T-TMR-TA29, see Table S1 in the Supporting Information) was obtained by the substitution of the 15th base C (cytosine) of TA29 for an internally labeled T (thymine), which is marked as C15T. C15T is a distant site of TA29 from the associated thrombin in the protein-aptamer complex and has five $G$ bases surrounded within its 4 nt region. ${ }^{20,21}$ However, both the 3'-end and 5'-end of the TA29 only have one $\mathrm{G}$ base within their $4 \mathrm{nt}$ regions. Among the three TA29 aptamers, only C15T-TMR-TA29 displays a significant reduction in FA $(\Delta r=-0.081)$ in the presence of human $\alpha$ thrombin with a molar ratio of 1:1 (Figure 1). In contrast, both 5'-TMR-TA29 $(\Delta r=0.010)$ and 3'-TMR-TA29 $(\Delta r=0.039)$ display an increase in FA (Figure 1). Interestingly, in response to the thrombin binding the unique labeling of TMR at C15T not only displays a significant reduction in FA but also causes the largest net FA change, which is $2.0-8.0$-fold as large as that obtained by using 5'-TMR-TA29 and 3'-TMR-TA29.

The observed FA changes of the three aptamers, which were caused by human $\alpha$-thrombin binding, were achieved in the presence of $100 \mathrm{mM} \mathrm{NaCl}$. We measured the affinity of the aptamers in the presence of $100 \mathrm{mM} \mathrm{NaCl}$ using capillary electrophoresis-laser induced fluorescence analysis. It was found that all three aptamers (C15T-TMR-TA29, 5'-TMRTA29, and 3'-TMR-TA29) maintained high affinity $\left(K_{\mathrm{d}}=0.6-\right.$ $1.0 \mathrm{nM}$, see Table S2 in the Supporting Information). This is consistent with previous work showing that the labeling of TMR did not significantly change the affinity of these aptamers. $^{20}$

We further investigated the effects of three metal ions $\left(\mathrm{Na}^{+}\right.$, $\mathrm{Mg}^{2+}$, and $\mathrm{Ca}^{2+}$ ) on the FA change of C15T-TMR-TA29 upon thrombin binding. As shown in Figure 2, the FA reduction of C15T-TMR-TA29 caused by thrombin binding is effectively aided by metal ion $\mathrm{Na}^{+}$. The maximum FA reduction was

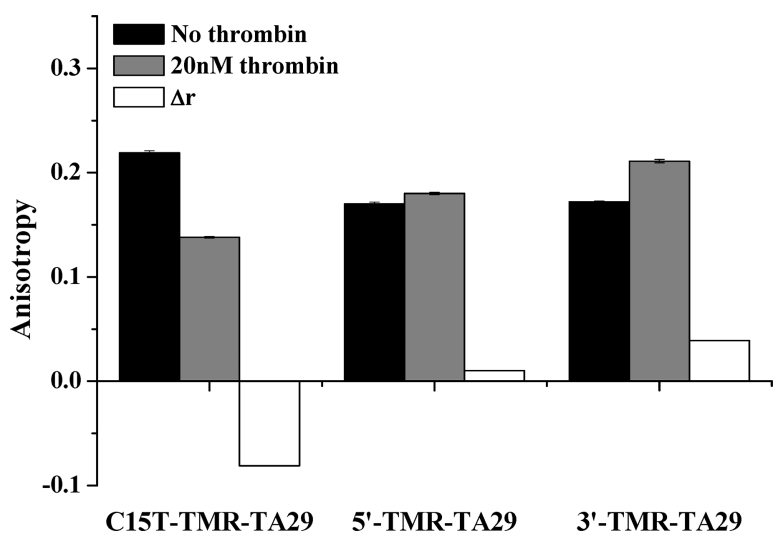

Figure 1. Fluorescence anisotropy (FA) of $20 \mathrm{nM}$ TA29 aptamers with a TMR label at a different position (C15T-TMR-TA29, 5'-TMRTA29, 3'-TMR-TA29) in the absence (black bars) and presence (gray bars) of $20 \mathrm{nM}$ thrombin and the fluorescence anisotropy change $(\Delta r$, white bars) of the corresponding aptamers. The buffer of $10 \mathrm{mM}$ Tris$\mathrm{HCl}, \mathrm{pH} 7.5,100 \mathrm{mM} \mathrm{NaCl}$ was used for the binding and measurement.

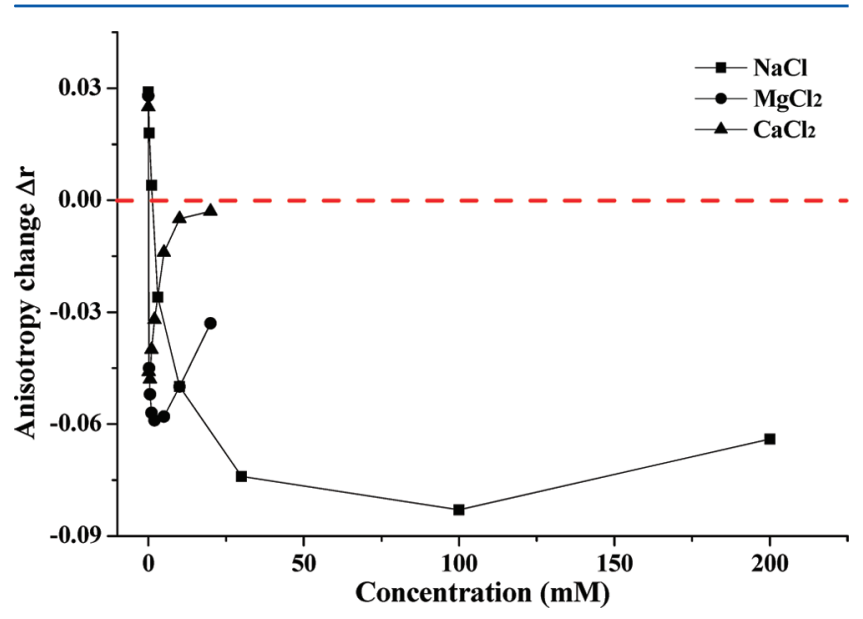

Figure 2. Fluorescence anisotropy change $\left(\Delta r=r-r_{\mathrm{f}}\right)$ of $20 \mathrm{nM}$ C15T-TMR-TA29 upon binding of $20 \mathrm{nM}$ thrombin at varying concentrations of $\mathrm{NaCl}$ (black squares), $\mathrm{MgCl}_{2}$ (black circles), and $\mathrm{CaCl}_{2}$ (black triangles). $r_{\mathrm{f}}$ and $r$ are the fluorescence anisotropy values in the absence and presence of thrombin, respectively.

obtained at $100 \mathrm{mM} \mathrm{NaCl}(\Delta r=-0.081)$. In contrast, FA of the C15T-TMR-TA29 $(\Delta r=0.029)$ slightly increases after its binding to thrombin in the absence of $\mathrm{Na}^{+}$. Similarly, the FA reduction upon thrombin binding can also be aided by $\mathrm{Mg}^{2+}$ and $\mathrm{Ca}^{2+}$ (Figure 2); however, the achieved maximum FA reductions obtained by $\mathrm{Mg}^{2+}(\Delta r=-0.059)$ and by $\mathrm{Ca}^{2+}(\Delta r=$ $-0.048)$ are lower than that obtained by $\mathrm{Na}^{+}(\Delta r=-0.081)$.

The FA change caused by thrombin binding is also affected by the type of the labeled fluorophore (Figure 3). To understand the effects of the labeled fluorophore, we investigated four fluorophores (TMR, FAM (6-carboxyfluorescein), $\mathrm{Cy} 3$, and $\mathrm{Cy} 5$ ). All the investigated fluorophores were singly labeled at the same site of TA29 (C15T). In addition to TMR, the labeled FAM can also generate a significant FA reduction in response to thrombin binding. Interestingly, this FAM-labeled C15T-TA29 requires the involvement of $\mathrm{Mg}^{2+}$ to achieve the maximum FA reduction $(\Delta r=-0.022)$. The use of the other two fluorophores, $\mathrm{Cy} 3$ and $\mathrm{Cy} 5$, cannot exhibit a FA reduction upon thrombin binding, probably due to a lack of a 

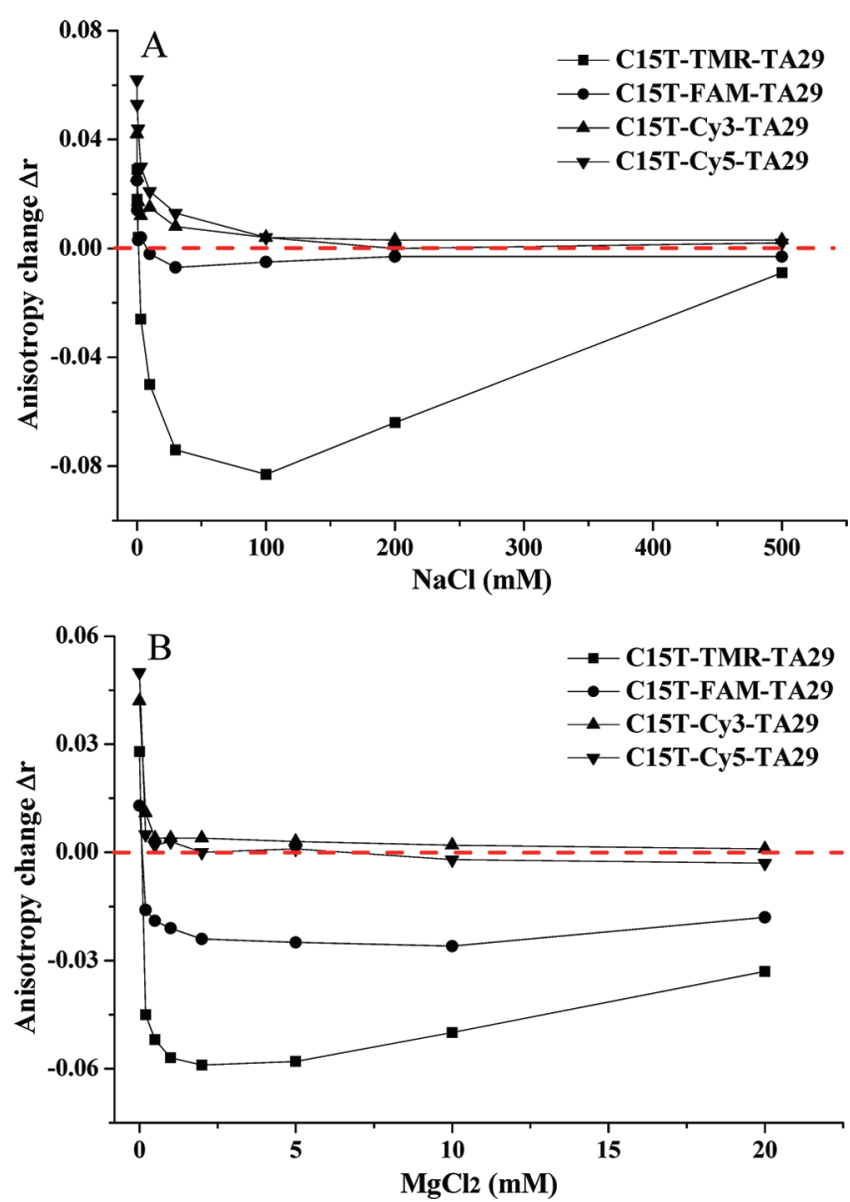

Figure 3. Fluorescence anisotropy change of $20 \mathrm{nM}$ thrombin aptamer labeled at $\mathrm{C} 15 \mathrm{~T}$ with TMR (black squares), FAM (black circles), Cy3 (black upward triangles), and Cy5 (black downward triangles) upon binding of $20 \mathrm{nM}$ thrombin in the presence of different concentration of $\mathrm{NaCl}$ (A) and $\mathrm{MgCl}_{2}$ (B). Binding buffer conditions: $10 \mathrm{mM}$ Tris$\mathrm{HCl}, \mathrm{pH}$ 7.5.

significant change in intramolecular interactions of the labeled Cy3/Cy5 upon protein binding.

In the observed FA reduction, it is critical that the intramolecular interactions between the labeled fluorophore (e.g., TMR and FAM) and the adjacent bases (mainly guanines) of the fluorescent aptamer occur and then they are diminished upon thrombin binding. This speculation is consistent with the observation of the thrombin bindinginduced FA reduction of C15T-TMR-TA29 $(\Delta r=-0.081)$, which indicates an increase in local rotation of the labeled TMR after the binding of the fluorescent aptamer to the protein and supports the hypothesized change in intramolecular interactions.

In contrast to that of the two aptamers $(r=0.170-0.172$ for 3'- and 5'-TMR-TA29), the unbound C15T-TMR-TA29 displays the largest FA value $(r=0.219)$. The results also prove the occurrence of the intramolecular interactions between the labeled TMR of C15T-TMR-TA29 and surrounded bases prior to the target protein binding, which can restrict the diffusional rotation of the labeled TMR and cause higher FA response.

The occurrence and elimination of the intramolecular interactions between the labeled fluorophore and the adjacent bases are also supported by fluorescence intensity analysis. It is known that the intramolecular interactions of the fluorophore labeled in ssDNA is often accompanied by a photoinduced electron transfer (PET) between the fluorophore (acceptor) and guanine bases (donor), which can cause an effective fluorescence quenching. The PET between a fluorophore and a guanine donor usually occurs in the strong coupling range at a distance of $0.07-1.0 \mathrm{~nm},{ }^{27}$ which is much less than that of Föster type resonance energy (with a critical distance of 2.0$8.0 \mathrm{~nm}) .{ }^{26}$ Among four bases of DNA (A, T, G, C), only guanine can act as an electron donor to a fluorophore acceptor and has high quenching efficiency. ${ }^{23}$ Upon protein binding, the quenched fluorescence of the C15T-TMR-TA29 can be restored (Figure $\mathrm{S} 1$ in the Supporting Information). The fluorescence of the partly quenched C15T-TMR-TA29 is 1.41.9-fold enhanced by an addition of the target protein at a molecular ratio of 1:1. In contrast, the fluorescence of 5 '- and 3'-TMR-TA29 just slightly increases by $50-54 \%$ and $22-36 \%$, respectively.

Taken together, the occurrence of the intramolecular interactions of the labeled fluorophore within the aptamer probe prior to the protein binding and the followed diminishment upon the protein binding are very critical to the development of a FA reduction approach for detection of protein binding.

To investigate whether the FA reduction is specific for target protein binding, we tested three types of proteins, including abundant proteins (bovine blood hemoglobin $(\mathrm{Hb})$, bovine serum albumin (BSA), human serum albumin (HSA), lactate dehydrogenase (LDH), and mouse immunoglobulin $\mathrm{G}(\mathrm{IgG})$ ), basic proteins (chicken egg lysozyme and bovine heart cytochrome C (CytC)), and thrombin-like proteins (prothrombin, Factor IX, ${ }^{28}$ and trypsin). All the tested proteins cannot cause any significant change in FA for both 3'-TMRTA29 and C15T-TMR-TA29 even in the presence of 25-fold excess proteins (Figure 4A,B).

To understand the benefits of the FA reduction, we further tested one known DNA binding protein, E. coli mismatch repair protein MutS. As shown in Figure $4 \mathrm{C}$, this protein can bind both aptamers (3'-TMR-TA29 and C15T-TMR-TA29) and cause an increase in FA of the two aptamers $(\Delta r=0.096$ for C15T-TMR-TA29; $\Delta r=0.053$ for 3'-TMR-TA29). In the tested MutS-aptamer system (100 nM MutS and $20 \mathrm{nM}$ aptamer), the target protein ( $20 \mathrm{nM}$ human $\alpha$-thrombin) was then added. Again, it was observed that the increased FA value for C15T-TMR-TA29 reduced from $r=0.316$ to $r=0.171(\Delta r$ $=-0.145$ ) by the addition of the target protein (human $\alpha$ thrombin). However, negligible FA change was observed for 3'TMR-TA29 $(\Delta r=0.001)$ while the same concentration of the target protein was added (Figure $4 \mathrm{C}$ ). These results suggest that FA reduction of C15T-TMR-TA29 is highly specific against the target protein binding and even resistant to the known DNA binding activity.

Both 3'-TMR-TA29 and C15T-TMR-TA29 display a thrombin concentration-dependent FA change in a standard buffer system (10 mM Tris- $\mathrm{HCl}, \mathrm{pH} 7.5,100 \mathrm{mM} \mathrm{NaCl})$, but C15T-TMR-TA29 displays a 1-fold larger FA change in magnitude than 3'-TMR-TA29 over the titrated concentration range of the thrombin (Figure 5A). On the basis of the signalto-noise ratio of $S / N>3$, the estimated low limits of detection (LOD) for the thrombin using the two fluorescent aptamers are comparable (LOD, $100 \mathrm{pM}$ ), and the estimated dynamic range is about $0.1-2 \mathrm{nM}$.

We further investigated the sensitivity and specificity of the FA assays in a complex medium. Surprisingly, 3'-TMR-TA29 

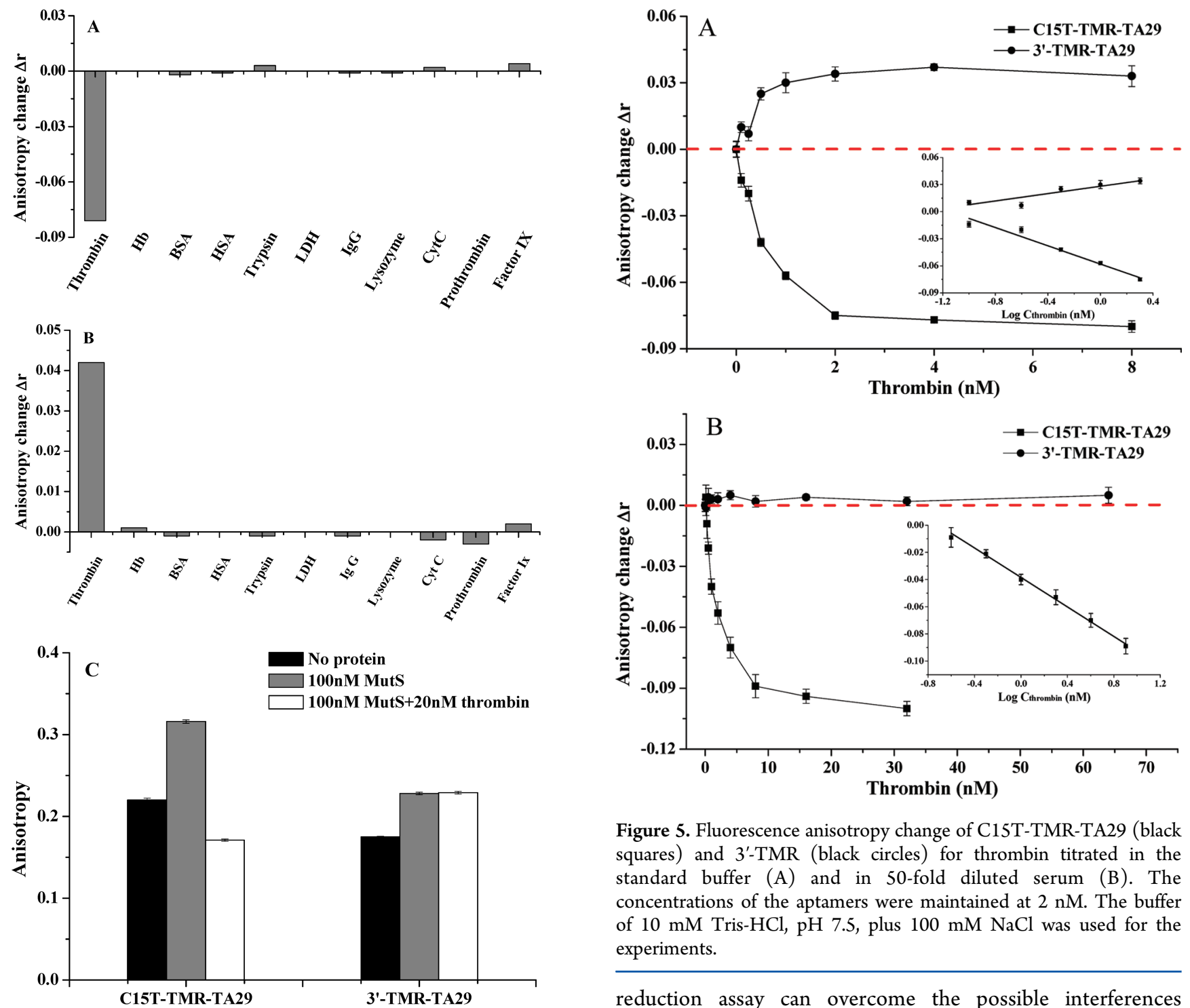

Figure 5. Fluorescence anisotropy change of C15T-TMR-TA29 (black squares) and 3'-TMR (black circles) for thrombin titrated in the standard buffer (A) and in 50-fold diluted serum (B). The concentrations of the aptamers were maintained at $2 \mathrm{nM}$. The buffer of $10 \mathrm{mM}$ Tris- $\mathrm{HCl}, \mathrm{pH} 7.5$, plus $100 \mathrm{mM} \mathrm{NaCl}$ was used for the experiments.

reduction assay can overcome the possible interferences resulting from nonspecific protein binding, strong fluorescence background, and high FA response of human serum itself. The estimated LOD is about $250 \mathrm{pM}$ in the presence of 50 -fold diluted human serum, and the estimated dynamic range is about $250 \mathrm{pM}-8 \mathrm{nM}$. In contrast, in a traditional molecular size increase-dependent FA assay using 3'-TMR-TA29 (Figure $5 B)$, the thrombin spiked in diluted serum failed to induce a reliable FA change even at $64 \mathrm{nM}$. Evidently, the developed FA reduction approach shows at least 256 times higher sensitivity.

In addition to the screened FA-sensitive antihuman $\alpha$ thrombin aptamer (C15T-TMR-TA29), a fluorescent aptamer was identified for detection of the PDGF-B chain using the FA reduction approach. The identified aptamer (5'-TMR-A1T-20t4 , see Table $S 1$ in the Supporting Information) was screened out from the modification of a previously known anti-PDGF-B chain aptamer 20t (5'-TGG GAG GGC GCG TTC TTC GTG GTT ACT TTT AGT CCC G-3'), which has a secondary structure motif of a three-way helix junction and a threenucleotide loop at the branch point. ${ }^{30}$ The fluorescent aptamer was obtained by truncating the 5 '-terminal four bases of the original 20t aptamer ${ }^{30-32}$ and substituting the first adenine (from the 5 ' end) for thymine in the truncated sequence and was fluorescently labeled by TMR at the $5^{\prime}$ end (5'-TMR-A1T20t-4). The FA response of the screened aptamer decreases 
when the fluorescent aptamer titrated with PDGF-BB (Figure S3 in the Supporting Information). Although the maximum FA reduction of 5'-TMR-A1T-20t-4 caused by PDGF-BB binding is not as large as that of C15T-TMR-TA29 obtained by thrombin $(\Delta r=-0.081)$, the results at least suggest that the FA reduction approach is potentially generalizable.

This work demonstrates a novel aptamer based FA approach for protein signaling, in which the $F A$ value decreases in response to the target protein binding. In the previously designed aptamer biosensors, the aptamer-protein binding (e.g., 3'-TMR-TA29) always induces an increased FA response. As spiked in protein-rich matrix (e.g., serum), conventionally labeled aptamer (e.g., 3'-TMR-TA29) cannot sense the target protein thrombin even at the concentration of $64 \mathrm{nM}$. Benefiting from its tolerance to nonspecific binding, the newly designed aptamer can be used to detect $250 \mathrm{pM}$ thrombin spiked in dilute serum, showing a dramatically enhanced specificity. It is evident that the new strategy brings up more promising practical applications for protein signaling.

In addition to the enhanced sensitivity and specificity, the developed FA reduction approach possesses all the advantages of the traditional FA assays compared with other protein signaling approaches. ${ }^{33,34}$ First, only a single fluorescent label is required per aptamer. Second, since it is a rationing technique (measuring the relative intensity of horizontal and vertical fluorescence emissions), FA measurement is inherently selfreferencing and independent of the variable fluorescence intensity. ${ }^{35}$ Because of this property, FA measurement overcomes the common problems raised in fluorescence intensity based assays, such as photobleaching, ${ }^{15}$ and is insensitive to source fluctuation and electronic drift. Third, FA analysis is a mix-and-measure method, simple and rapid, and has potentials in high-throughput analysis.

\section{ASSOCIATED CONTENT}

\section{S Supporting Information}

Experimental section and supporting materials. This material is available free of charge via the Internet at http://pubs.acs.org.

\section{AUTHOR INFORMATION}

\section{Corresponding Author}

*Phone: 86-10-62849600. Fax: 86-10-62849600. E-mail: hlwang@rcees.ac.cn.

\section{Notes}

The authors declare no competing financial interest.

\section{ACKNOWLEDGMENTS}

This work was supported by the grants from the National Natural Science Foundation of China (Grants 21077129, 20877091, 20890112, 21125523, and 20921063) and the National Basic Research Program of China (Grants 2009CB421605 and 2010CB933502) to Dr. H. Wang.

\section{REFERENCES}

(1) Wilson, D. S.; Szostak, J. W. Annu. Rev. Biochem. 1999, 68, 611647.

(2) Cho, E. J.; Lee, J.-W.; Ellington, A. D. Annu. Rev. Anal. Chem. 2009, 2, 241-264.

(3) Nimjee, S. M.; Rusconi, C. P.; Sullenger, B. A. Annu. Rev. Med. 2005, 56, 555-583.

(4) Bouchard, P. R.; Hutabarat, R. M.; Thompson, K. M. Annu. Rev. Pharmacol. Toxicol. 2010, 50, 237-257.
(5) Gold, L.; Ayers, D.; Bertino, J.; Bock, A.; et al. PLoS One 2010, 5, e15004.

(6) Ostroff, R. M.; Bigbee, W. L.; Franklin, W.; Gold, L.; Mehan, M.; et al. PLoS One 2010, 5, e15003.

(7) Jayasena, S. D. Clin. Chem. 1999, 45, 1628-1650.

(8) Liu, J.; Cao, Z.; Lu, Y. Chem. Rev. 2009, 109, 1948-1998.

(9) Tombelli, S.; Minunni, M.; Mascini, M. Biosens. Bioelectron. 2005, 20, 2424-2434.

(10) Song, S.; Wang, L.; Li, L.; Zhao, J.; Fan, C. Trends Anal. Chem. 2008, 27, 108-117.

(11) Potyrailo, R. A.; Conrad, R. C.; Ellington, A. D.; Hieftje, G. M. Anal. Chem. 1998, 70, 3419-3425.

(12) McCauley, T. G.; Hamaguchi, N.; Stanton, M. Anal. Biochem. 2003, 319, 244-250.

(13) Li, W.; Wang, K.; Tan, W.; Ma, C.; Yang, X. Analyst 2007, 132, 107-113.

(14) Cao, Z.; Tan, W. Chem.-Eur. J. 2005, 11, 4502-4508.

(15) Fang, X.; Cao, Z.; Beck, T.; Tan, W. Anal. Chem. 2001, 73, $5752-5757$

(16) Gokulrangan, G.; Unruh, J. R.; Holub, D. F.; Ingram, B.; Johnson, C. K.; Wilson, G. S. Anal. Chem. 2005, 77, 1963-1970.

(17) Deng, T.; Li, J.; Zhang, L.-L.; Jiang, J.-H.; Chen, J.-N.; Shen, G.L.; Yu, R.-Q. Biosens. Bioelectron. 2010, 25, 1587-1591.

(18) Lakowicz, J. R. Principles of Fluorescence Spectroscopy, 3rd ed.; Springer-Verlag: Berlin, Heidelberg, Germany, 2006.

(19) Vallée-Bélisle, A.; Plaxco, K. W. Curr. Opin. Struct. Biol. 2010, 20, 518-526.

(20) Zhang, D.; Lu, M.; Wang, H. J. Am. Chem. Soc. 2011, 133, 9188-9191.

(21) Tasset, D. M.; Kubik, M. F.; Steiner, W. J. Mol. Biol. 1997, 272, 688-698.

(22) Edman, L.; Mets, U.; Rigler, R. Proc. Natl. Acad. Sci. U.S.A. 1996, 93, 6710-6715.

(23) Torimura, M.; Kurata, S.; Yamada, K.; Yokomaku, T.; Kamagata, Y.; Kanagawa, T.; Kurane, R. Anal. Sci. 2001, 17, 155-160.

(24) Wang, W.; Chen, C.; Qian, M. X.; Zhao, X. S. Sens. Actuators, B: Chem. 2008, 129, 211-217.

(25) Maruyama, T.; Shinohara, T.; Ichinose, H.; Kitaoka, M.; Okamura, N.; Kamiya, N.; Goto, M. Biotechnol. Lett. 2005, 27, 13491354.

(26) Heinlein, T.; Knemeyer, J.-P.; Piestert, O.; Sauer, M. J. Phys. Chem. B 2003, 107, 7957-7964.

(27) Qu, P.; Chen, X.; Zhou, X.; Li, X.; Zhao, X. Sci. China Ser. B: Chem. 2009, 52, 1653-1659.

(28) Hamaguchi, N.; Ellington, A.; Stanton, M. Anal. Biochem. 2001, 294, 126-131.

(29) Millioni, R.; Tolin, S.; Puricelli, L.; Sbrignadello, S.; Fadini, G. P.; Tessari, P.; Arrigoni, G. PLoS One 2011, 6, e19603.

(30) Green, L. S.; Jellinek, D.; Jenison, R.; Heldin, C.-H.; Janjie, N. Biochemistry 1996, 35, 14413-14424.

(31) Zhang, H.; Li, X. F.; Le, X. C. J. Am. Chem. Soc. 2008, 130, 3435 .

(32) Zhang, H.; Li, X. F.; Le, X. C. Anal. Chem. 2009, 81, 77957800.

(33) Gradinaru, C. C.; Marushchak, D. O.; Samim, M.; Krull, U. J. Analyst 2010, 135, 452-459.

(34) Jameson, D. M.; Ross, J. A. Chem. Rev. 2010, 110, 2685-2708. (35) Chen, X.; Levine, L.; Kwok, P. Y. Genome Res. 1999, 9, 492498. 